\title{
PHOTOLUMINESCENCE OF ZnO NANOSTRUCTURE PREPARED BY CATALYST - ASSISTED VAPOR - LIQUID - SOLID TECHNIQUE
}

\author{
Tran Thi Quynh Hoa, Ta Dinh Canh, Nguyen Ngoc Long*, \\ Nguyen Viet Tuyen, and Nguyen Duy Phuong \\ Faculty of Physics, Hanoi University of Science, Hanoi \\ 334 Nguyen Trai, Thanh Xuan, Hanoi, Vietnam
}

Received 27 November 2006

\begin{abstract}
$\mathrm{ZnO}$ semiconductor nanostructures have been synthesized by thermal evaporation of mixture of $\mathrm{ZnO}$ and graphite powders. The thin layer of gold coated on Si substrates was used as a catalyst. The structure analysis shows high crystallinity of $\mathrm{ZnO}$, their preferred orientation along the $\left(\begin{array}{ll}0 & 0\end{array}\right.$ 2) plane of the wurtzite phase and their chemical purity. The scanning electron microscopy (SEM) images of products indicate that $\mathrm{ZnO}$ nanowires have a diameter of about $40-150 \mathrm{~nm}$ and a length of up to tens of micrometers. The morphology and structure of $\mathrm{ZnO}$ nanowires and nanorods depend on the thicknesses of the Au layers. The fact that Au nanoparticles are located at the tips of the nanowires represents a strong evidence for a growth process dominated by the vapor - liquid - solid mechanism. The low temperature photoluminescence spectra of the $\mathrm{ZnO}$ nanowires indicate a group of the ultraviolet narrow peaks and a blue - green very broad peak at $500 \mathrm{~nm}$.
\end{abstract}

Keywords: Photoluminescence, $\mathrm{ZnO}$ nanostructure, vapor-liquid-solid mechanism

\section{INTRODUCTION}

Zinc oxide $(\mathrm{ZnO})$ has been one of the most promising oxide semiconductor materials because of its good optical, electrical, piezoelectric properties and potential application. Numerous studies on the preparations and physical properties of $\mathrm{ZnO}$ films and, specially, nanoparticles have been reported.

Nanowires have been successfully fabricated by using various approaches including the vapor transport process, chemical vapor deposition, electrochemical deposition technique, and sol - gel etc.

In this work, we report on the fabrication, mechanism and features of $\mathrm{ZnO}$ nanowires using a vapor transport process via the vapor - liquid - solid mechanism. The origin of the luminescent peaks will be discussed.

\section{EXPERIMENTS}

$\mathrm{ZnO}$ nanostructures were synthesized by means of a vapor transport process on $\mathrm{Si}$ substrates ( $\mathrm{n}$ -

\footnotetext{
*Corresponding author e-mail: longnn@vnu.edu.vn
} 
type, $\left(\begin{array}{lll}1 & 0 & 0\end{array}\right)$ ) by using an $\mathrm{Au}$ as a catalyst. The Si substrates were ultrasonically cleaned in acetone for $30 \mathrm{~min}$. The cleaned Si substrates were coated with a layer of Au using a sputtering technique. Source materials are mixture of $\mathrm{ZnO}$ and $\mathrm{C}$ powder (molar ratio is 1:0.1). Arrangement of experiment is shown in Fig. 1. The flow rate of argon was $500 \mathrm{sccm}$. The furnace was heated up to $1000^{\circ} \mathrm{C}$ and kept at the temperature for $60 \mathrm{~min}$. After reaction at the temperature range of $500-800^{\circ} \mathrm{C}$, the substrate surface was coated by white wax - like materials.

The crystal structure, composition of the $\mathrm{ZnO}$ nanostructures was analyzed by $\mathrm{X}$ - ray diffraction (XRD Bruker - AXS D5005) and energy dispersive X - ray analysis (EDX; Oxford Isis 300). The morphology of the $\mathrm{ZnO}$ nanostructure was characterized by scanning electron microscopy (SEM; JEOL - JSM 5410 LV). The photoluminescence measurement was done by using a spectrofluorometer (FL3 - 22 Jobin Yvon Spex, USA).

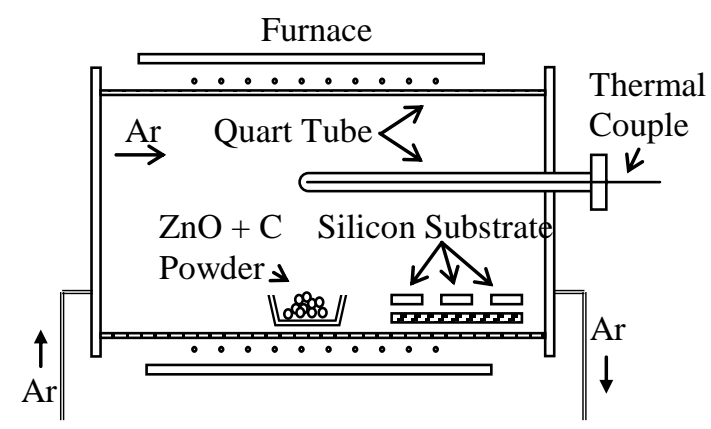

Fig. 1: Experimental set up for synthesis of $\mathrm{ZnO}$ nanostructures

\section{RESULTS AND DISCUSSION}

XRD patterns of $\mathrm{ZnO}$ products are given in Fig. 2. Most of the samples indicated a growth along the $\left(\begin{array}{lll}0 & 0 & 2\end{array}\right)$ direction. No peaks of $\mathrm{Zn}$, but two peaks of $\mathrm{Au}\left(\begin{array}{lll}1 & 1 & 1\end{array}\right)$ and $\left(\begin{array}{lll}2 & 0 & 0\end{array}\right)$ have been detected in the XRD patterns.

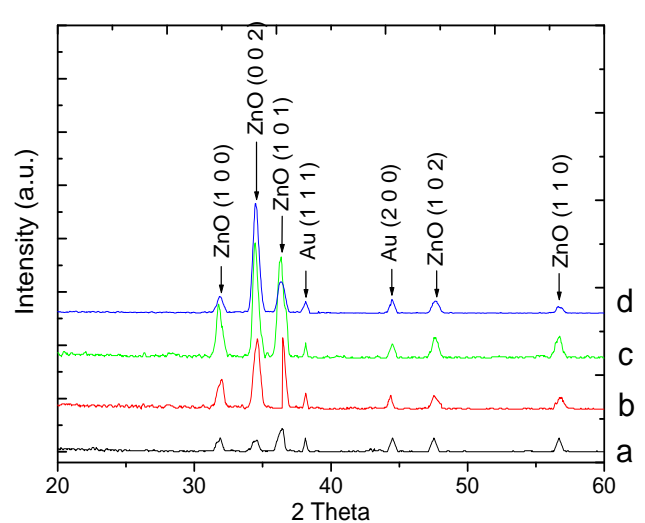

Fig. 2: XRD patterns of $\mathrm{ZnO}$ nanostructures on substrates coated with Au layers of $25 \mathrm{~nm}$ $(a, b) ; 50 \mathrm{~nm}(c) ; 100 \mathrm{~nm}(d)$ in thickness



Fig. 3: EDX spectrum of $\mathrm{ZnO}$ nanowires on substrate with an Au layer of $25 \mathrm{~nm}$ in thickness 
Figure 3 shows the EDX pattern of the $\mathrm{ZnO}$ nanowires, showing peaks due to $\mathrm{Zn}$, O. The EDX spectrum clearly indicates that the nanostructures consist of pure $\mathrm{ZnO}$.

The thickness of Au films played an important role in the growth of the nanowires. It was observed that when the thickness of the Au film increased from 25 to $100 \mathrm{~nm}$, the diameter of the wires is increased as shown in Fig. 4.

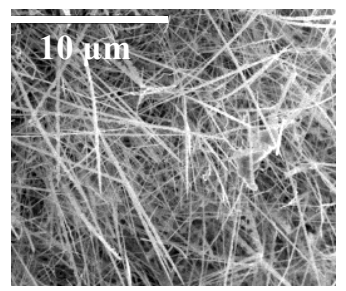

(a)

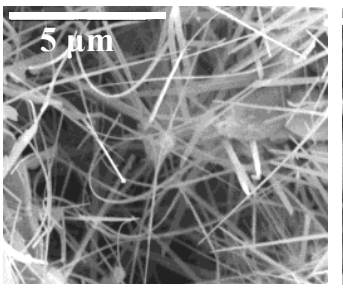

(b)

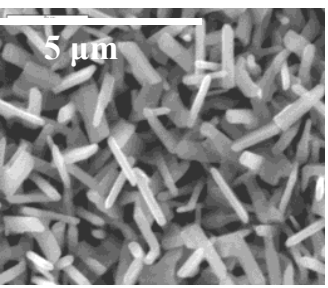

(c)

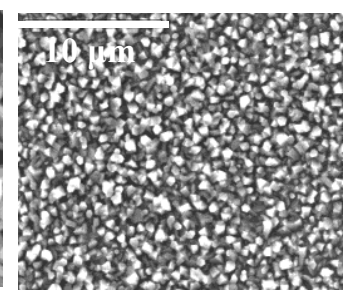

(d)

Fig. 4: SEM of ZnO nanostructures deposited on substrates coated with Au layers of different thicknesses: $(a, b) 25 \mathrm{~nm}$, (c) $50 \mathrm{~nm}$ and (d) $100 \mathrm{~nm}$

Figure 5 illustrates SEM images of $\mathrm{ZnO}$ nanowires ending with spherical nanoparticles. The presence of spherical nanoparticles at the end of the nanowires represents strong evidence for a growth process dominated by the VLS mechanism (Fig. 6). In the present work, carbon was used for reducing sublimate temperature of $\mathrm{ZnO}$. The $\mathrm{Zn}$ and $\mathrm{ZnO}_{1-\mathrm{x}}$ vapor is deposited on the $\mathrm{Si}$ substrate located downstream, and reacted with the Au catalyst to form $\mathrm{Au}-\mathrm{ZnO}$ alloy droplets.

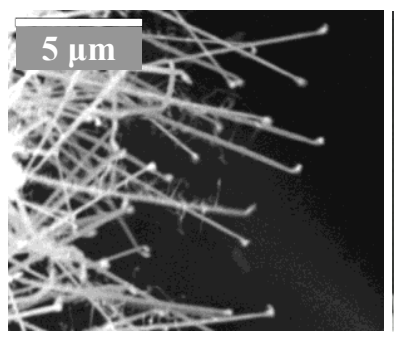

(a)

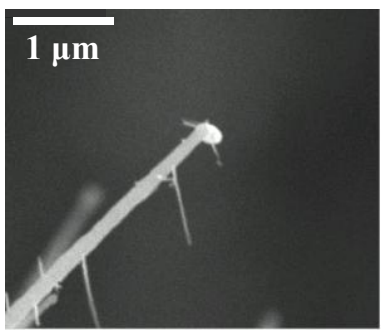

(b)

Fig. 5: SEM images of ZnO nanowires

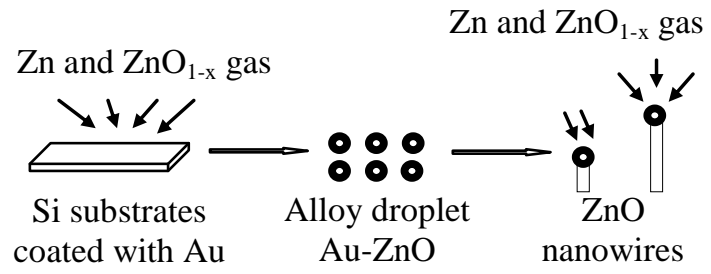

Fig. 6: Mechanism of the growth of nanowires, nanorods

As the droplets become supersaturated in $\mathrm{Zn}$ and $\mathrm{ZnO}_{1-\mathrm{x}}$, $\mathrm{ZnO}$ nanostructures are formed. The size and shape of the nanocomposite $\mathrm{Au}-\mathrm{ZnO}$ droplets directly control the diameter of the 1D nanostructure. Smaller diameter of the Au droplets favors the growth of thinner nanowires, whereas larger Au droplets help to grow thicker nanorods. 


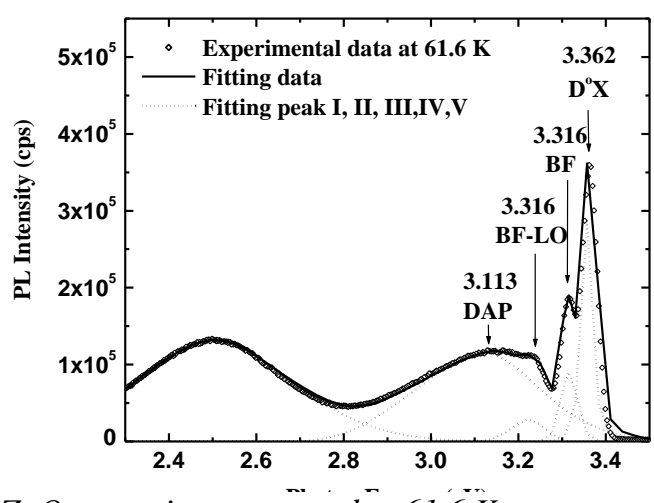

Fig. 7: PL spectrum of $\mathrm{ZnO}$ nanowires measured at $61.6 \mathrm{~K}$

The photoluminescence (PL) measurements at low temperature were done for the $\mathrm{ZnO}$ nanowires (Fig. 7). Most of PL spectra have a blue - green broad peak and a group of ultraviolet (UV) narrow peaks.

The blue - green emission band was commonly attributed to a deep - level (oxygen vacancies, interstitial zinc), trap-state or surface state emission. Because, even at low temperature, $\mathrm{ZnO}$ is a nonstoichiometric oxide and presents deep defects such as oxygen vacancies $\left(V_{o}^{0}\right)$, interstitial zinc $\left(Z n_{i}^{0}\right)$, ionized oxygen vacancies $\left(V_{O}^{+}\right)$, ionized interstitial zinc $\left(Z n_{i}^{+}\right)[1]$.

In this work, we concentrate on the investigation and explanation of the origin of UV emission peaks. PL spectra of the $\mathrm{ZnO}$ nanostructure measured in the temperature range from $15 \mathrm{~K}$ to 300 $\mathrm{K}$ are shown in Fig. 8.

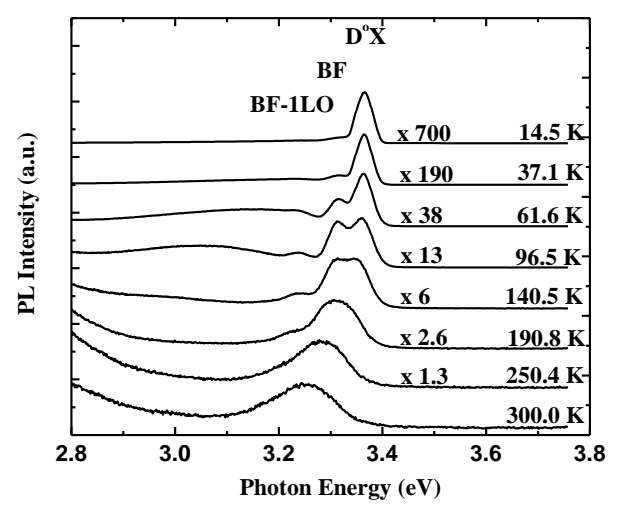

Fig. 8: The low temperature PL spectra of $\mathrm{ZnO}$ nanowires

It can be seen that the PL spectra exhibited one broad peak at about $3.113 \mathrm{eV}$ (peak IV) and three narrow peaks (III, II, I) at around $3.243 \mathrm{eV}, 3.316 \mathrm{eV}, 3.362 \mathrm{eV}$, respectively. As the temperature increases, these peaks are shifted toward the low energy side, while their intensity decreases. Peak I disappeared at temperature approximate to $166.1 \mathrm{~K}$, whereas two peaks II, III exist until room temperature.

In order to clarify the origins of emission peaks, the experimental data were fitted by symmetric Pearson VII function (reliability factors $>99.3 \%$ ). 


$$
y=A \frac{2 \sqrt{m} \cdot e^{\Gamma\left(2^{1 / m}-1\right)}}{\pi w_{h} e^{\Gamma(m-0.5)}}\left[1+4 \frac{2^{1 / m}-1}{w_{h}^{2}}\left(x-x_{C}\right)^{2}\right]^{-m}
$$

where $A$ is integrated intensity, $w_{h}$ is full width at half maximum (FWHM), $m$ is peak flack parameter, $x_{C}$ is peak position, and $\Gamma$ is function defined as:

$$
\Gamma(a)=\int_{0}^{\infty} t^{a-1} e^{-x} d t
$$

From the fitted results, we have found the position, integrated intensity and FWHM of UV peaks.

Fig. 9 shows the position of these peaks as a function of temperature. As it can be seen from this figure, the experimental values of peak I are fitted rather well to the Varshni's curve calculated by using Varshni's semiempirical formula [2]:

$$
E(T)=E(0)-\frac{\alpha T^{2}}{T+\beta}
$$

where $\mathrm{E}(0)$ is position peak at $0 \mathrm{~K}, \alpha$ and $\beta$ are fitting parameters.

By curve fitting the experimental data in Fig. 10, we found that $\alpha=-9.8 \times 10^{-4} \mathrm{eV} / \mathrm{K}, \beta=-800$ $\mathrm{K}$ and $E(0)=3.369,3.324$ and $3.251 \mathrm{eV}$ for peaks I, II, III, respectively.

Based on fitting results, peak I can be ascribed to a neutral donor - bound exciton (denoted by $\left.\mathrm{D}^{\circ} \mathrm{X}\right)$.

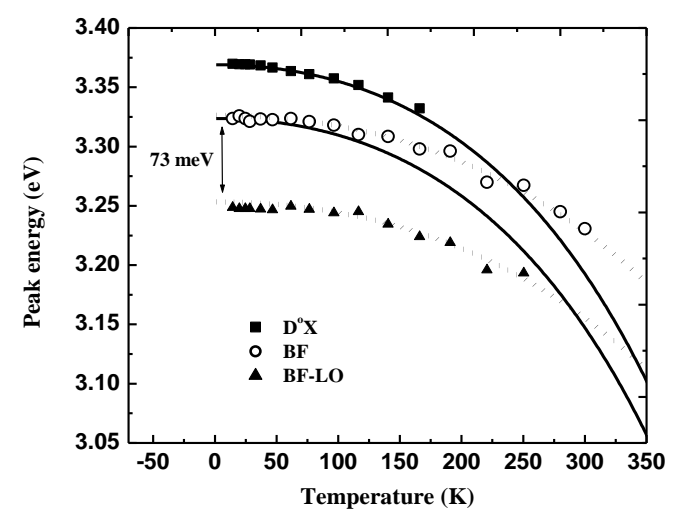

Fig. 9: The observed peak energy of UV peak as a function of temperature

Peak II is still maintained up to room temperature, and its position varies with temperature more slowly than the energy band gap. Thus, this peak is attributed to the recombination of bound charge carriers on impurities with free carriers in the allowed bands (BF). In our case, the sample is an $\mathrm{n}$ - type semiconductor, so peak II can be the result of recombination between an electron bound on a donor and a free hole in the valence band (BF) [3]. The energy distance between peaks II and III at different temperatures is about $73 \mathrm{meV}$, this value is approximate to long optical (LO) - phonon energy of $72 \mathrm{meV}$. Hence, peak III can be attributed to the phonon replica of peak II (BF - LO).

Figure 10 shows the integrate PL intensity of $\mathrm{D}^{\circ} \mathrm{X}$ and $\mathrm{BF}$ peak as a function of temperature. It was found that the integrated PL intensity could be fitted by formula [4]: 


$$
I(T)=\frac{I(0)}{1+A e^{-\frac{E}{k_{B} T}}}
$$

where $I(0)$ is the emission intensity at $0 \mathrm{~K}, A$ is a constant, $E$ is activation energy of the thermal quenching process and $k_{B}$ is Boltzmann constant.

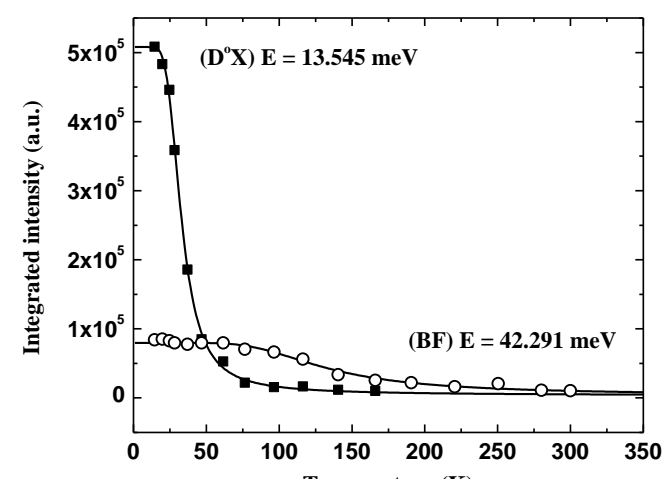

Fig. 10: The temperature dependence of integrated intensity of $D^{\circ} X$ and $B F$ peaks

By curve fitting the experimental data in Fig. 10, we found that $E=13.5 \mathrm{meV}$ and $42.3 \mathrm{meV}$ for $\mathrm{D}^{\circ} \mathrm{X}$ and $\mathrm{BF}$ peaks, respectively. The value of $13.5 \mathrm{meV}$ is close to the value of the binding energy of the exciton bound to the neutral donors, experimentally obtained to be $12 \mathrm{meV}$ [5]. The value of $42.3 \mathrm{meV}$ being larger than thermal activation energy at room temperature ( $~ 26$ $\mathrm{meV}$ ) indicates the possibility of existence of the BF peak at high temperatures.

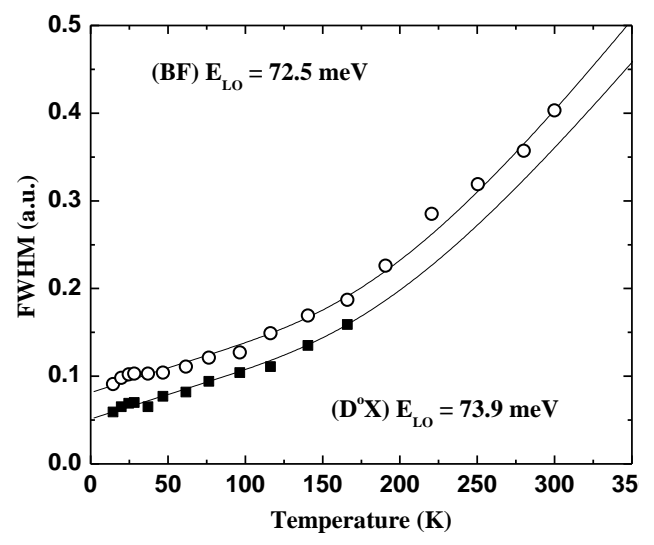

Fig. 11: The observed FWHM of $D^{\circ} X$ and BF peak as a function of temperature

FWHM $(\Gamma)$ of peak $\mathrm{D}^{\circ} \mathrm{X}, \mathrm{BF}$ as a function of temperature is shown in Fig. 11. It was found that FWHM could be fitted by following [6]:

$$
\Gamma(T)=\Gamma(0)+\Gamma_{L A} T+\frac{\Gamma_{L O}}{e^{\frac{E_{L O}}{k_{B} T}}-1}
$$

where $\Gamma(0)$ is the broadening parameter at $0 \mathrm{~K}, \Gamma_{L A}, \Gamma_{L O}$ are parameters measured by 
weighting of LA, LO phonons, respectively, and $E_{L O}$ is the energy of LO phonons.

From the fitting we obtain $E_{L O}=73.9$ and $72.5 \mathrm{meV}$ for $\mathrm{D}^{\circ} \mathrm{X}$ and BF peak, respectively. Such values are in good agreement with the previously reported value of $72 \mathrm{meV}$.

The broad peak at about $3.113 \mathrm{eV}$ (peak IV) is interpreted as a donor - acceptor pairs (DAP) emission. The position of DAP emission is described as:

$$
h v=E_{g}-\left(E_{A}+E_{D}\right)+\frac{q^{2}}{\varepsilon r}
$$

where $E_{g}$ is the band gap, $E_{A}$ and $E_{D}$ are the acceptor and donor binding energy, respectively, $q$ is the electrical charge of the acceptor and donor ions, $\varepsilon$ is the dielectric constant, and $\mathrm{r}$ is the donor - acceptor distance.

With increasing temperature, carriers on donor-acceptor pairs with small distance $r$ are released into the band, which results in extinguishing the high-energy side of DAP emission line, and the line is shifted to the low-energy side as observed in our experiment.

\section{CONCLUSIONS}

The $\mathrm{ZnO}$ nanowires have been fabricated by controlling the conditions of the vapor - phase transport. The $\mathrm{ZnO}$ nanowires with diameter of about $40-150 \mathrm{~nm}$ and length to tens of micrometer show the typical wurtzite crystal structure. The growth process is explained by means of a VLS mechanism. The PL spectra of $\mathrm{ZnO}$ nanowires in the near band edge region were investigated in temperature range from $14.5 \mathrm{~K}$ to $300 \mathrm{~K}$. Four UV emission peaks were attributed to neutral donor - bound exciton $\left(\mathrm{D}^{\circ} \mathrm{X}\right)$, recombination of an electron bound on donor with a free hole in the valence band (BF), phonon replica of the peak BF with one LO - phonon energy (BF-LO), donor - acceptor pairs (DAP).

\section{ACKNOWLEDGEMENT}

This work is supported by Natural Science Council, Ministry of Science and Technology of Vietnam (Project $\mathrm{N}^{\mathrm{o}}$ 811304). Authors of this paper would like to express their sincere gratitude to the Center for Materials Science (CMS), Faculty of Physics, University of Science, Hanoi National University for permission to use equipment.

\section{REFERENCES}

1. Gupta, T.K.J. (1990), Am. Ceram. Soc., vol. 73(7), p. 1817.

2. Varshni, Y.P. (1967), Physica (Amsterdam), vol. 34, p. 149.

3. Chi, D.H., Binh, L.T.T., Binh, N.T., Khanh, L.D., and Long, N.N. (2006), Appl. Surf. Scien., vol. 252, p. 2770.

4. Holtz, P.O., Monemar, B., and Lozykowski, H.J. (1985), Phys. Rev., vol. B 32, p. 986.

5. Ko, H.J., Chen, Y.F., Zhu, Z., Yao, T., Kobayashi, I., and Uchiki, H. (2000), Appl. Phys. Lett., vol. 76, p. 1905.

6. Makino, T., Chia, C.H., Tuan, N.N., Segawa, Y., Kawasaki, M., Ohtomo, A., Tamura, K., and Koinuma, H. (2000), Appl. Phys. Lett., vol. 76, p. 349. 